\title{
Role of Catalysts on Electrode Activity in Oxygen Diffusion Hybrid Polymer Electrode
}

\author{
Miki Kawashima, Yoshikazu Sato, ${ }^{*}$ Mineo Sato, \\ and Masakazu SAKAGUCHI \\ Department of Active Material Chemistry, Division of Fundamental Science and Technology, \\ Graduate School of Science and Technology, Niigata University, \\ Niigata 950-21, Japan \\ * Department of Applied Chemistry, Faculty of Engineering, Niigata University, \\ Niigata 950-21, Japan
}

(Received July 20, 1990)

\begin{abstract}
A film type of gas diffusion hybrid polymer (GDHP) electrode with about $100 \mu \mathrm{m}$ in thickness was prepared by the electrochemical polymerization of pyrrole in the poly(vinyl chloride) binder polymer film which contained a metal-phthalocyanine (M-Pc; $\mathrm{M}$ : $\mathrm{Co}, \mathrm{Cu}$, or Pd) crystal as a catalyst. The role of the catalyst on the oxygen reduction reaction in the GDHP electrode was investigated. There were some correlations between the electrode activity and the dispersion state of the crystalline particles of the catalyst. The GDHP electrode with Co-Pc catalyst gave a high activity, and was stable for a long time working. An optimum amount of Co-Pc catalyst for the electrode activity was found to exist around $50 \mathrm{wt} \%$ to the amount of polymer matrix (binder polymer and catalyst). By using a tritium tracer method, it was also found that the permeability of water vapor through the electrode took a maximum value when using the electrode with the optimum amount of $\mathrm{Co}-\mathrm{Pc}$ catalyst. The reaction mechanism of this film type GDHP electrode was considered on the basis of the behavior of the reaction species.
\end{abstract}

KEY WORDS Hybrid Polymer Film / Gas Diffusion Electrode / Electrochemical Oxygen Reduction / Polypyrrole / Metal Phthalocyanine / Poly(vinyl chloride) / Tritium Tracer Method /

Conventional gas diffusion electrodes were difficult to keep high activity during a long time working without leak of the electrolyte through the electrode, because of their porous structure. Many people have contrived some methods to control the wettability of the electrode, e.g., the construction of a double-layered electrodes, ${ }^{1,2}$ the selection of carbon black carriers with adequate hydrophobicity, ${ }^{3,4}$ etc. There are another practical problems. For example, the aggregation and coming-off of the catalyst from the carbon carrier were sometimes encountered during a long operation, ${ }^{5-7}$ because the polytetrafluoroethylene used as a binder can not fix the catalyst tightly.

We have already presented a method to prepare two types, plate type and film type, of a gas diffusion hybrid polymer (GDHP) electrode composed of conductive polypyrrole (PPy), polyvinylchloride (PVC) binder polymer and metal phthalocyanine (M-Pc) ${ }^{8}$ In the work, the activity of the GDHP electrodes have been found to be dependent on the oxygen permeability of binder polymer. From the result, it has been estimated that the reaction spices, such as oxygen, water molecule, and $\mathrm{OH}^{-}$ion, dissolve in the GDHP electrode bulk. Thus, the oxygen reduction reaction by using the GDHP electrode is taken place without forming the three phase interface. In order to clarify the reaction mechanism in the GDHP electrode, it is necessary to investigate the behavior of the permeation of reacting species and the dispersion state of the catalyst in the 
electrode. The film type of GDHP electrode should be more favorable for this investigation than the plate type because one can ignore the additional factor, that is, the behavior of the carbon paper supporter in the plate type electrode. In this study the film type electrode was, therefore, prepared.

Three kinds of crystalline M-Pc were used as the catalyst for electrochemical reduction of oxygen. The surface area and the grain size distribution of the M-Pc particles are thought to be major factors to control the electrode activity. Also, the progress in the reaction should require the transfer of $\mathrm{OH}^{-}$, which could be intimately correlative with the path way of water molecules. We have already confirmed that a radioisotope tracer method is very effective to investigate the mass transfer in the electrode separating gas and electrolyte..$^{3,4,9,10}$ By means of a tritium tracer method, we were able to investigate the relationship between the electrode activity and the permeability of water vapor through the electrode film.

\section{EXPERIMENTAL}

\section{Materials}

Poly(vinyl chloride) (PVC) (SUMIRIT EX13, molecular weight; 80,000, Sumitomo Kagaku Co., Ltd.) was used as received. $N, N$ Dimethylformamide (DMF) (reagent grade, Wako Pure Chemical Industries Ltd.) was used without further purification. Pyrrole (Py) (Tokyo Kasei Kogyo Co., Ltd.) was distilled under a reduced pressure and stored in a nitrogen atmosphere. Tetra- $n$-butylammonium perchlorate (TBAP) (Tokyo Kasei Kogyo Co., Ltd.) was purified by the recrystallization from the mixed solvent of ether and acetone. Acetonitrile (AN) (Wako Pure Chemical Industries Ltd.) was reagent grade. Co-Pc was prepared by the reaction of cobalt dichloride (Tokyo Kasei Kogyo Co., Ltd.) with isophthalonitrile (Tokyo Kasei Kogyo Co., Ltd.) in 1,8-diazabicyclo[5.4.0]-7-undecene (Wako
Pure Chemical Industries Ltd.) by refluxing in nitrobenzene at $210^{\circ} \mathrm{C}$ for $6 \mathrm{~h}$. Pd-Pc and $\mathrm{Cu}-\mathrm{Pc}$ were prepared from paradium dichloride and copper dichloride, respectively, in the same method as that for $\mathrm{Co}-\mathrm{Pc}$, the refluxing condition of which was in $n$-amylalchole at $130^{\circ} \mathrm{C}$ for $6 \mathrm{~h}$.

\section{Preparation of GDHP Electrode}

The film type of GDHP electrode was prepared by the same method as reported previously. ${ }^{8}$ PVC was used as a binder polymer because of its high affinity for PPy as well as of its high facility to form a thin film. $\mathrm{Co}-\mathrm{Pc}, \mathrm{Cu}-\mathrm{Pc}$, and $\mathrm{Pd}-\mathrm{Pc}$ were used as the catalyst for oxygen reduction reaction. The three kinds of GDHP electrodes with different grain sizes of the catalyst were prepared as follows; the grain size of the catalyst were adjusted by grinding the blended powder of M-Pc and PVC with an agate mortar for $5 \mathrm{~min}, 10 \mathrm{~min}$, and $30 \mathrm{~min}$, and referred as sample- $a$, sample-b, and sample-c in the order of the grinding time interval, respectively. The ratio of $\mathrm{Co}-\mathrm{Pc}$ to $\mathrm{PVC}$ was $1: 1$ in weight. The ground powder $(2 \mathrm{~g})$ was added into DMF $(10 \mathrm{ml})$, then dispersed by stirring for $30 \mathrm{~min}$ at $50^{\circ} \mathrm{C}$ on a magnetic stirrer. The electrochemical polymerization of Py was carried out with a polymer matrix film obtained by casting the dispersed solution on an ITO glass plate. ${ }^{11,12}$ The electrolyte consisting of TBAP and $0.1 \mathrm{M}$ Py solution of $\mathrm{AN}$ was used in the electrochemical polymerization. The charge density for the reaction was $2.5 \mathrm{C} \cdot \mathrm{cm}^{-2}$ under a constant potential, $2.0 \mathrm{~V}$ vs. $\mathrm{Ag} / \mathrm{AgNO}_{3}$ $\left(0.01 \mathrm{~N} \quad \mathrm{AgNO}_{3}\right)$. The film prepared was detached from the ITO glass, washed adequately by $\mathrm{AN}$ and dried at $50^{\circ} \mathrm{C}$ in vacuo, and then used as a GDHP electrode.

\section{Characterization of Catalyst}

Surface area of M-Pc catalyst before grinding was determined by a BET method using ethylene as an adsorbate gas at $-183^{\circ} \mathrm{C}$. The dispersion state and the crystal structure 
of catalyst embedded in the electrode film were examined by a transmitted scanning electron microscope (Electron Probe Microanalyzer EPMA-8705, Shimadzu Co., Ltd.) and an X-ray diffractometer (Geigerflex RAD-rA, Rigaku Denki Co., Ltd.), respectively.

\section{Measurement}

The electrical conductivity of the GDHP electrode film was measured by a conventional four-prove method. The thickness of the electrode film was measured by a micrometer (Digital Micrometer $\mu$-mate M-30, SONY Magnescale Inc.). The characteristics of the electrode films used in this study are summarized in Table I.

The apparatus for measuring the electrode activity and the permeability of water vapor was the same as the apparatus reported previously. ${ }^{8,10}$ The electrode activity was evaluated by the polarization measurement at $25^{\circ} \mathrm{C}$. In particular the current density at $-340 \mathrm{mV}$ (vs. $\mathrm{Hg} / \mathrm{HgO}$ ) was adopted in this study as a standard estimate for the output of the electrode. The water vapor permeating through the electrode film was measured by means of the tritium tracer method. The electrolyte used for this measurement was an aqueous $\mathrm{KOH}$ solution $(1 \mathrm{M})$ labeled by tritium. The specific radioactivity (about $4000 \mathrm{~Bq} \mathrm{~cm}^{-3}$ ) of the tritiated aqueous solution was high enough to detect the small amount of water vapor. The surface of the electrolyte was covered by liquid paraffin to prevent the evaporation of water vapor from the surface to the atmosphere and also to prevent the absorption of carbon dioxide from the atmosphere. Therefore, the water vapor evaporating from the electrolyte permeated through the electrode film and then mixed with unreacted oxygen gas, being absorbed into a liquid scintillator (PCS-1, Amersham International Co., Ltd.) in the vial. The amount absorbed of the water vapor was determined by applying an isotope dilution method for the radioactivity detected with a liquid scintillation
Table I. Characteristics of electrode films

\begin{tabular}{|c|c|c|c|}
\hline & \multirow{2}{*}{ Sample } & \multirow{2}{*}{$\begin{array}{c}\text { Thickness } \\
\qquad \mu \mathrm{m}\end{array}$} & \multirow{2}{*}{$\frac{\text { Conductivity }}{\mathrm{S} \mathrm{cm}^{-1}}$} \\
\hline & & & \\
\hline \multirow[t]{3}{*}{$\mathrm{Co}-\mathrm{Pc}$} & Sample-a & 115 & 0.015 \\
\hline & Sample-b & 112 & 0.013 \\
\hline & Sample-c & 110 & 0.011 \\
\hline \multirow[t]{3}{*}{$\mathrm{Pd}-\mathrm{Pc}$} & Sample-a & 100 & 0.005 \\
\hline & Sample-b & 98 & - \\
\hline & Sample-c & 98 & - \\
\hline \multirow[t]{3}{*}{$\mathrm{Cu}-\mathrm{Pc}$} & Sample-a & 52 & 1.01 \\
\hline & Sample-b & 50 & - \\
\hline & Sample-c & 50 & 0.77 \\
\hline
\end{tabular}

system (LSC-703, Aloka Co., Ltd.). ${ }^{10}$

\section{RESULTS AND DISCUSSION}

Figure 1 shows the SEM image of three kinds of $\mathrm{M}-\mathrm{Pc}(\mathrm{M}=\mathrm{Co}, \mathrm{Cu}, \mathrm{Pd})$ crystalline powder used as a catalyst, and the surface area of them is listed in Table II. The $\mathrm{Co}-\mathrm{Pc}$ catalyst consists of small crystalline particles with $c a .5 \mu \mathrm{m} \times 1 \mu \mathrm{m}$ in size, while the primary crystalline particles of $\mathrm{Pd}-\mathrm{Pc}$ and $\mathrm{Cu}-\mathrm{Pc}$ catalysts are very fine and form secondary particle crowds. These characteristics of catalysts are reflected in the degree of their BET surface area.

The relationship between the electrode activity and the dispersion state of the catalyst in the electrode film was investigated for three types of GDHP electrodes having different grain sizes of the catalyst. The dispersion state of Co-Pc catalyst in the electrode film is shown in Figure 2. It can be seen that the catalyst was the most dispersive in the sample-c. X-Ray diffraction analysis for these films exhibited that, before and after grinding, there were neither change in the crystal structure nor change in the size of crystalline particles of the catalyst. Therefore, it seems that the grinding of the catalyst results in break-down of the secondary particle crowds to primary crystalline particles.

The permeability of water through the 


\section{$5 \mu \mathrm{m}$}

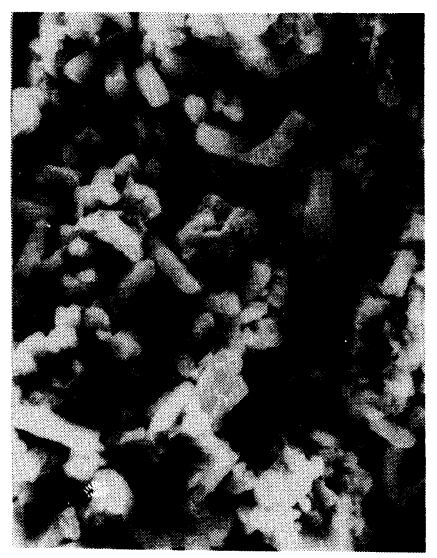

Co-Pc

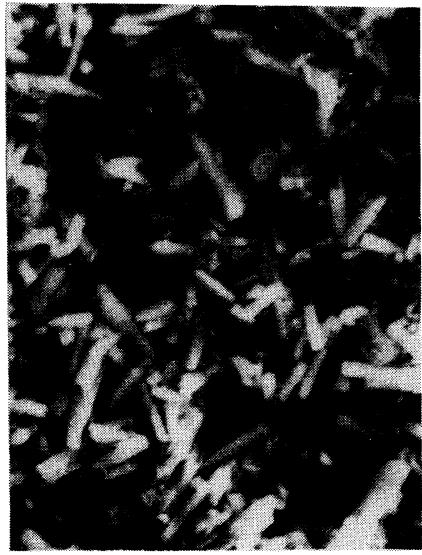

Cu-Pc

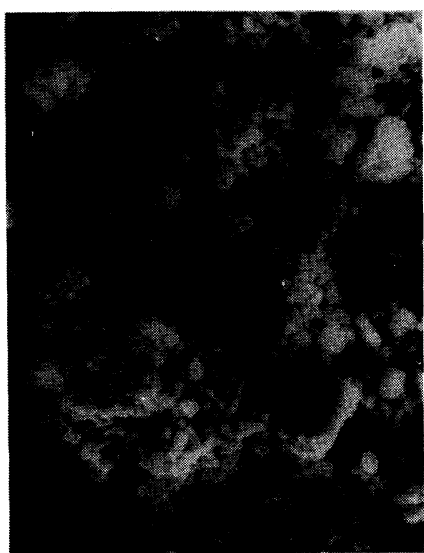

Pd-Pc

Figure 1. SEM image of $\mathrm{M}-\mathrm{Pc}(\mathrm{M}=\mathrm{Co}, \mathrm{Cu}, \mathrm{Pd})$ powder.

Table II. BET surface area of M-Pc powder used as catalyst for GDHP electrode

\begin{tabular}{cc}
\hline M-Pc & BET surface area $/ \mathrm{m}^{2} \mathrm{~g}^{-1}$ \\
\hline $\mathrm{Co}-\mathrm{Pc}$ & 3.00 \\
$\mathrm{Cu}-\mathrm{Pc}$ & 8.03 \\
$\mathrm{Pd}-\mathrm{Pc}$ & 9.61
\end{tabular}

GDHP electrode film was measured. Figure 3 shows a typical relationship between the permeability of water vapor and the reaction time. A plot of the permeability against square root time was found to be linear. The result implies that the permeation of water vapor occurs via diffusion process. From the experiment, however, it can not be concluded whether water molecule dissolves in the binder polymer or water vapor penetrates into the electrode film. Probably, it is impossible to clarify the state of water in the electrode under working conditions. It should be understood that the slope of the straight line shown in Figure 3 represents the degree of a driving force for water permeation through the electrode film. The slope is, therefore, expressed as the permeation character of water vapor in the following.

The electrode activity and the permeation character of water vapor are shown in Figure 4 for the electrode with $\mathrm{Co}-\mathrm{Pc}$ catalyst. There seems to be an optimum grinding condition of catalyst for higher activities among the GDHP electrodes with the same composition. Sampleb which showed the highest electrode activity maintained a stable electrode potential for about $1000 \mathrm{~h}$ under a constant current density, $0.05 \mathrm{~mA}$. The permeation character of water vapor corresponds to the behavior of the electrode activity. The boundary between Co-Pc particles and PVC matrix would be extended by the treatment of grinding the catalyst, resulting in an increase in number of the diffusion pathway of water molecule as well as an increase in reaction site. Generally, water molecules in polymer films serve as a carrier for diffusion of $\mathrm{OH}^{-}$ion. ${ }^{11}$ However, further grinding of the catalyst may make the network of water molecule pathway cut into shreds. Since the water molecules mainly thread their way through the PVC bulk in such a case, the 


\section{$\longmapsto$ \\ 100 um}

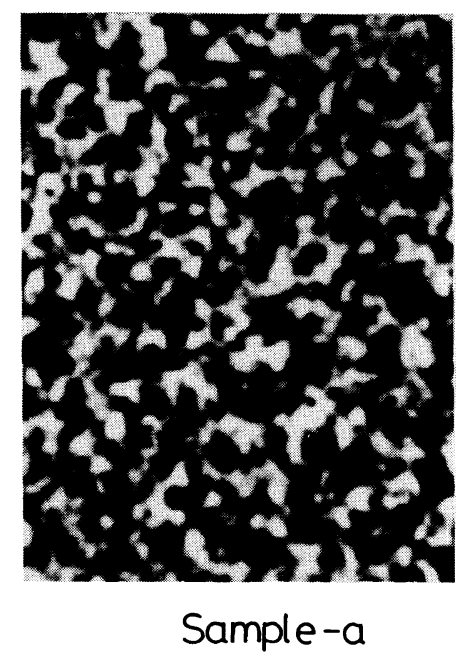

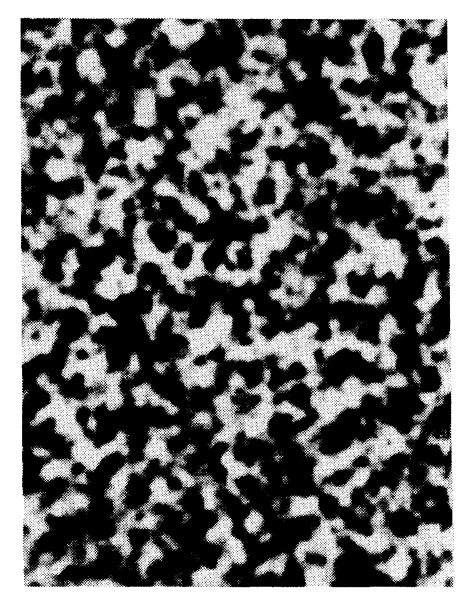

Sample-b

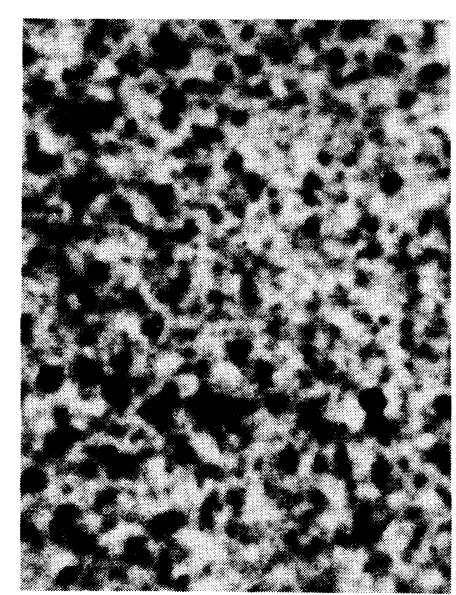

Sample-c

Figure 2. Dispersion state of Co-Pc catalyst in film type of GDHP electrode with composition of $\mathrm{Co}-\mathrm{Pc} / \mathrm{PVC} / \mathrm{PPy}(\mathrm{Co}-\mathrm{Pc}: \mathrm{PVC}=1: 1$ in weight ratio).

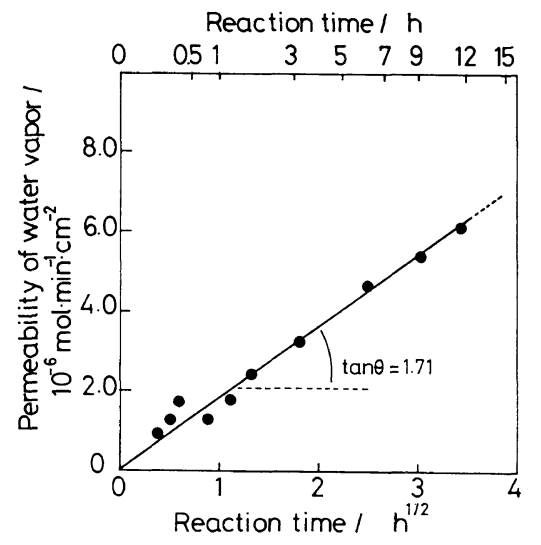

Figure 3. Relationship between permeability of water vapor and reaction time for film type of GDHP electrode with composition of $\mathrm{Co}-\mathrm{Pc} / \mathrm{PVC} / \mathrm{PPy}(\mathrm{Co}-\mathrm{Pc}: \mathrm{PVC}=1: 1$ in weight ratio).

diffusion of $\mathrm{OH}^{-}$ion becomes lower and then the electrode activity falls down.

Figure 5 shows the dispersion state of $\mathrm{Cu}-\mathrm{Pc}$ catalyst. $\mathrm{Cu}-\mathrm{Pc}$ catalyst seems to be dispersed with high density in the electrode, because of its small secondary particles as shown in Figure 1. In fact, the thickness of the electrode film

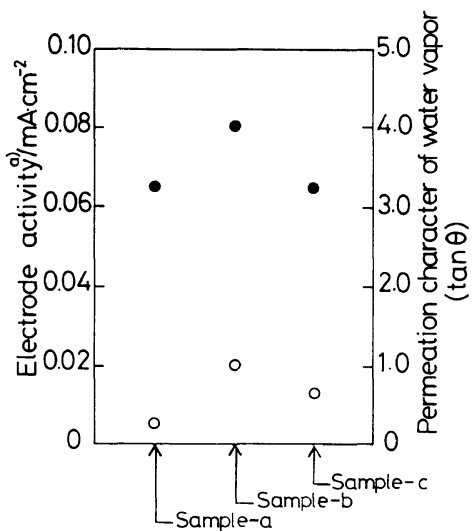

Figure 4. Effect of grain size of $\mathrm{Co}-\mathrm{Pc}$ catalyst on electrochemical characteristics of GDHP electrode with composition of $\mathrm{Co}-\mathrm{Pc} / \mathrm{PVC} / \mathrm{PPy}$ (Co-Pc: $\mathrm{PVC}=1: 1$ in weight ratio). represents electrode activity, and $\bigcirc$ represents permeation character of water vapor.

a) Electrode activity represents current density under $-340 \mathrm{mV}$ vs. $\mathrm{Hg} / \mathrm{HgO}$.

with $\mathrm{Cu}-\mathrm{Pc}$ catalyst is reduced to be about a half of that of the electrode film including the same amount of another catalysts (Table I). The diffusion rate of reaction species in the electrode should decrease due to the dispersion 


\section{$\vdash 1$ \\ 100 um}

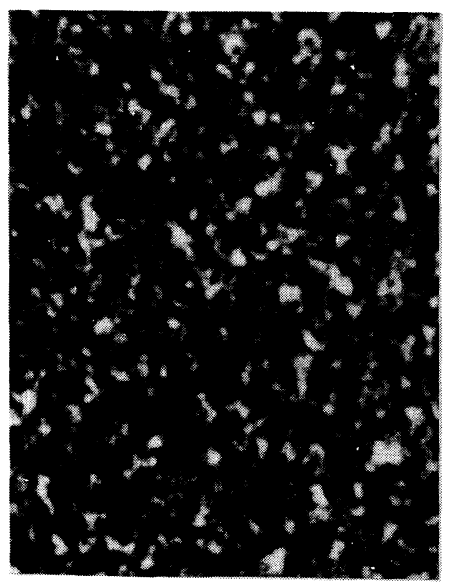

Sample-a

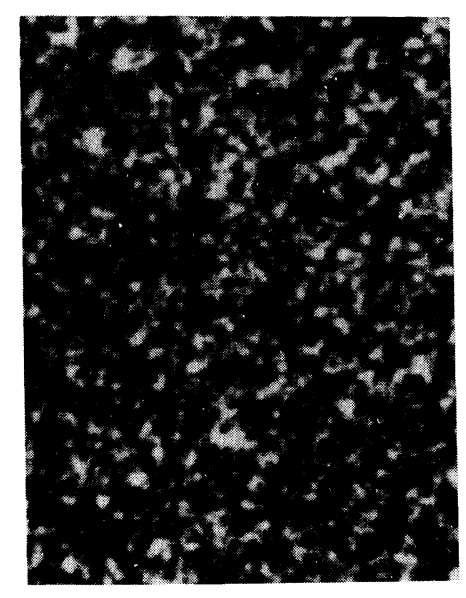

Sample-b

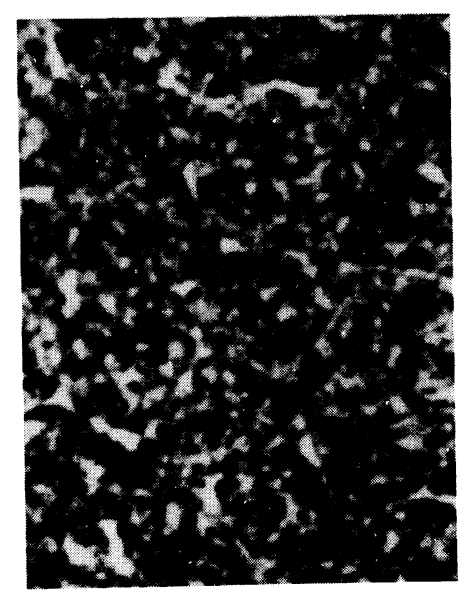

Sample-c

Figure 5. Dispersion state of $\mathrm{Cu}-\mathrm{Pc}$ catalyst in film type of GDHP electrode with composition of $\mathrm{Cu}-\mathrm{Pc} / \mathrm{PVC} / \mathrm{PPy}(\mathrm{Cu}-\mathrm{Pc}: \mathrm{PVC}=1: 1$ in weight ratio).

state with high density of $\mathrm{Cu}-\mathrm{Pc}$ catalyst, as shown evidently by the results for the permeation character of water vapor in Figure 6. In addition, the catalytic activity of $\mathrm{Cu}-\mathrm{Pc}$ for the oxygen reduction reaction is relatively low because of its high redox potential. ${ }^{14,15}$ Therefore, the GDHP electrode with $\mathrm{Cu}-\mathrm{Pc}$ catalyst had a lower electrode activity than that of the electrode with Co-Pc catalyst, in spite of its higher electrical conductivity (Table I).

The dispersion state of Pd-Pc catalyst in the electrode film is shown in Figure 7. The effect of grinding on the dispersion state of the catalyst can also be seen in this case. In particular, a more contrast image was obtained in the sample-c of $\mathrm{Pd}-\mathrm{Pc}$ catalyst than in the sample-c of $\mathrm{Co}-\mathrm{Pc}$ and $\mathrm{Cu}-\mathrm{Pc}$ catalysts although crystalline particles were well ground in the film. As shown in Figure 8, both of the electrode activity and the permeation character of water vapor are found to be higher than those in the case of $\mathrm{Co}-\mathrm{Pc}$ catalyst. The size of crystalline particles of $\mathrm{Pd}-\mathrm{Pc}$ is smaller than that of $\mathrm{Co}-\mathrm{Pc}$ as shown in Figure 1. The smaller is the size of crystalline particles, the stronger

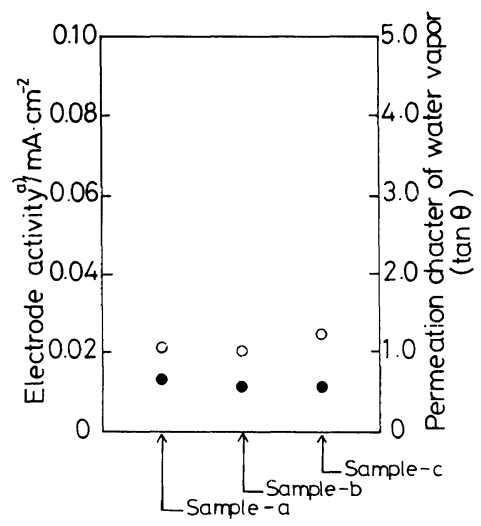

Figure 6. Effect of grain size of $\mathrm{Cu}-\mathrm{Pc}$ catalyst on electrochemical characteristics of GDHP electrode with composition of $\mathrm{Cu}-\mathrm{Pc} / \mathrm{PVC} / \mathrm{PPy}(\mathrm{Cu}-\mathrm{Pc}: \mathrm{PVC}=1: 1$ in weight ratio). represents electrode activity, and $O$ represents permeation character of water vapor.

a) Electrode activity represents current density under $-340 \mathrm{mV}$ vs. $\mathrm{Hg} / \mathrm{HgO}$.

becomes the adhesion force for secondary aggregation of crystalline particles. Thus, the effect of the grain size of the catalyst on the electrode activity appears more clearly in the case of Pd-Pc catalyst. However, the electrolyte 

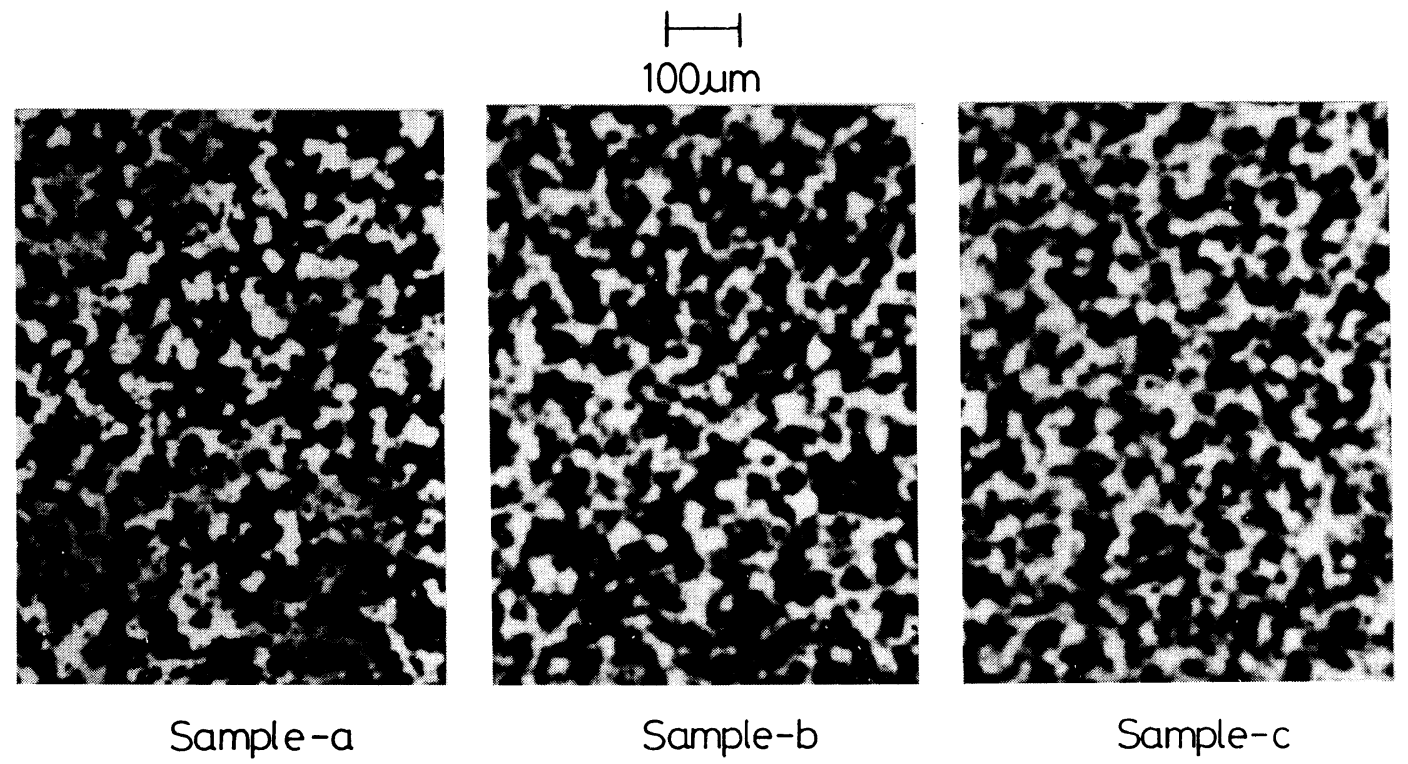

Figure 7. Dispersion state of Pd-Pc catalyst in film type of GDHP electrode with composition of $\mathrm{Pd}-\mathrm{Pc} / \mathrm{PVC} / \mathrm{PPy}(\mathrm{Pd}-\mathrm{Pc}: \mathrm{PVC}=1: 1$ in weight ratio).

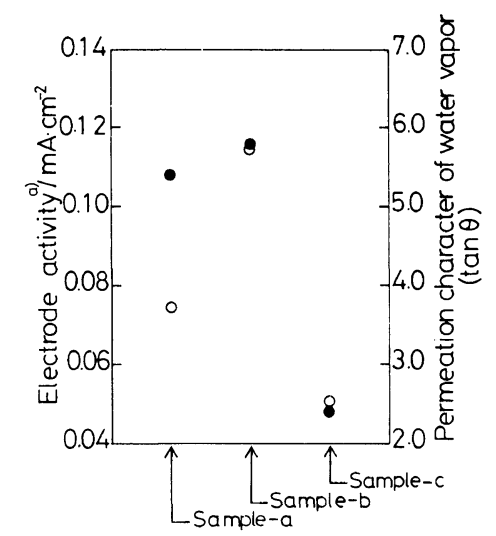

Figure 8. Effect of grain size of $\mathrm{Pd}-\mathrm{Pc}$ catalyst on electrochemical characteristics of GDHP electrode with composition of $\mathrm{Pd}-\mathrm{Pc} / \mathrm{PVC} / \mathrm{PPy}(\mathrm{Pd}-\mathrm{Pc}: \mathrm{PVC}=1: 1$ in weight ratio). represents electrode activity, and $\bigcirc$ represents permeation character of water vapor.

a) Electrode activity represents current density under $-340 \mathrm{mV}$ vs. $\mathrm{Hg} / \mathrm{HgO}$.

leak through the electrode film was found in all of the GDHP electrodes only with Pd-Pc catalyst, although the electrode activity was still maintained stable. This phenomenon may result from weak affinity of $\mathrm{Pd}-\mathrm{Pc}$ for PVC.
For conventional porous gas diffusion electrodes, it is known that the disappearance of activity necessarily accompanies a steep increase in the amount of the permeation of water vapor, indicating a decay of three phase equilibrium in the porous electrodes. ${ }^{3,4}$ This principle can not be applied to the GDHP electrode because three phase boundaries are not formed. It is supported that the reaction species dissolve and diffuse in the bulk of the GDHP electrode. Therefore, the fact that the electrode activity was maintained stable even in the situation of the electrolyte leak may suggests that oxygen permeability of the GDHP electrode film is similar to that of the electrolyte.

The effect of the amount of catalyst on the electrode activity was investigated for the GDHP electrode of sample-b with $\mathrm{Co}-\mathrm{Pc}$ (Figure 9). An optimum amount of $\mathrm{Co}-\mathrm{Pc}$ catalyst for the electrode activity was found to exist around $50 \mathrm{wt} \%$ to the amount of the polymer matrix. By using a tritium tracer method, it was also found that the permeability of water vapor through the electrode took a 


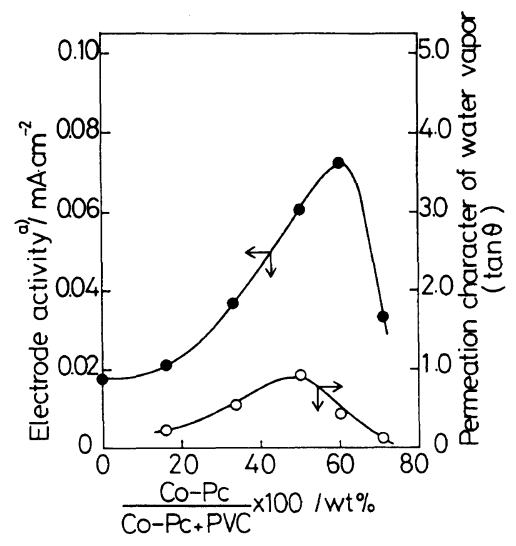

Figure 9. Dependence of amount of catalyst on electrochemical characteristics of GDHP electrode with composition of $\mathrm{Co}-\mathrm{Pc} / \mathrm{PVC} / \mathrm{PPy}$.

a) Electrode activity represents current density under $-340 \mathrm{mV}$ vs. $\mathrm{Hg} / \mathrm{HgO}$.

maximum value when using the electrode with the optimum amount of Co-Pc catalyst. It is worthy of notice that the activity for oxygen reduction reaction was observed for the GDHP electrode without any catalyst. This result suggests that the PPy itself has a little activity for oxygen reduction reaction. ${ }^{13}$ From the behavior of the permeation character of water vapor, the increase in the amount of the catalyst is thought to give not only an increase in the number of the reaction site in the GDHP electrode but also an increase in the number of the pathway for reaction species. However, when the amount of catalyst was more than $50 \mathrm{wt} \%$ to the amount of the polymer matrix, the electrode activity fell down. It can be considered that the decrease in number of the pathway in the PVC bulk, through which the reaction species can diffuse, lead to an appreciable decrease in the permeability of water vapor as shown in Figure 9. Therefore, the activity of the GDHP electrode should be controlled by the permeability of the reaction species, such as oxygen, water molecule and especially $\mathrm{OH}^{-}$ion.

Recently, Li et al. ${ }^{13}$ pointed out that a PPy film was relatively unstable in an alkaline solution. However, in the case of the GDHP electrode, the electrode activity was found to be stable for more than $1000 \mathrm{~h}$ under a constant current density of $0.05 \mathrm{~mA} \mathrm{~cm}^{-2}$. The discrepancy between these results may be attributed to the hybridization of PPy with PVC binder polymer. Further investigation should be needed to clarify the stability of the PPy in the GDHP electrode.

\section{CONCLUSION}

The activity of the film type of GDHP electrode was found to correspond to the degree of the driving force for water molecules to permeate through the electrode film in addition to the activity of the catalyst included in the electrode. The permeation phenomenon of water vapor was affected by both of the dispersion state of the catalyst particles and the amount of the catalyst in the GDHP electrode. In the present work, it was found that the existence of the catalyst in the GDHP electrode played not only a role of the catalysis for the oxygen reduction reaction but also an important role of controlling the permeation of the reaction species.

Acknowledgments. The author are indebted to Mr. Imai and Dr. H. Tanaka (both of them TOYO INK Mfg. Co., Ltd.) for their valuable discussion, and also to $\mathrm{Mr}$. $\mathrm{K}$. Uematsu (Niigata University) for electrochemical measurement, and finally to $\mathrm{Mr}$. M. Kobayashi (Niigata University) for SEM analysis.

\section{REFERENCES}

1. N. Kanaki, H. Hara, H. Shimada, and Y. Shiromoto, Kagakukogaku Ronbunshu, 6, 172 (1980).

2. Y. Matsuda, T. Nukuda, and M. Morita, Denki Kagaku, 51, 921 (1983).

3. Y. Sato, M. Sato, and M. Sakaguchi, Denki Kagaku, 57, 1000 (1989).

4. M. Sakaguchi, K. Uematsu, A. Sakata, Y. Sato, and M. Sato, Electrochimica Acta, 34, 625 (1989).

5. M. Watanabe, Shokubai, 31, 308 (1989). 
6. R. J. Remick, US DOE Rep, NO. DEC-NASA-208-4, 45 (1982).

7. R. J. Remick, US DOE Rep, NO. DEC-NASA-208-1, 26 (1981).

8. M. Kawashima, H. Tanaka, and M. Sakaguchi, $J$. Electroanal. Chem., 286, 123 (1990).

9. M. Sato, M. Ohta, and M. Sakaguchi, J. Electroanal. Chem., 277, 151 (1990).

10. M. Sakaguchi and M. Ohta, J. Electrochem. Soc., 136, 1923 (1989).

11. O. Niwa and T. Tamamura, J. Chem. Soc., Chem.
Commun., 817 (1984).

12. O. Niwa, M. Hikita, and T. Tamamura, Synth. Met., 18, 677 (1984).

13. Y. Li and R. Qian, Synth. Met., 26, 139 (1988).

14. F. H. Moser and A. L. Thomas, Ed., "The Phthalocyanine," Vol. 1, CRC Press, Boca Raton, Florida, 1983, p 81.

15. A. K. Shukla, R. Manoharan, C. P. Hammet, and J. B. Goodenough, J. Appl. Electrochem., 15, 774 (1985). 